\title{
PRODUCT VARIETY INDUCED COMPLEXITY AND ITS MEASUREMENT
}

\author{
Slavomir Bednar \\ Technical University of Kosice, Faculty of Manufacturing Technologies with a seat in Presov, Bayerova 1, 08001 \\ Presov, Slovak Republic, slavomir.bednar@tuke.sk \\ Vladimir Salanci \\ Technical University of Kosice, Faculty of Manufacturing Technologies with a seat in Presov, Bayerova 1, 08001 \\ Presov, Slovak Republic, vladimir.salanci@ centrum.sk
}

Keywords: complexity, combinatorics, configuration, product, scale

Abstract: Mass customization has become a novel trend in the manufacturing of products as it offers high product flexibility to customers. As a result, producers of such products have to cope with incresing demands on manufacturing and related complexity management. This paper aims to develop so called configuration complexity measure to determine the extent of mass-customized production variety. Product configuration complexity scale has been also developed in order to assess concurrent product architecturs based on the offered variety extent for their product. The method presented in this paper is a useful method with minimum input data required and with important output information for product decision-makers.

\section{Introduction}

High frequency of topics on complexity-related problems within mass customized (MC) production, including technology of production, in research publications clearly evokes their significance. Especially product variety induced complexity is often a matter of interest for theorists and practitioners. Our focus in this paper goes towards an exploration of product variety induced complexity (VIC) based on combinatorial principles to measures and to enumerate the VIC. Finally, in Section 3 we analyze mutual relations between numbers of possible product configurations and establish so called VIC scace to capture the effect of growing number of product components with impact on overall production complexity.

\section{Related work}

So far, several approaches have been taken in order to assess complexity of the manufacturing system. Different authors focused on partial problems - sources of complexity and covered only a partial manufacturing environment. Complexity of any system is affected by three variables, namely state of the system elements, their number and relationships among them [1]. Different definitions of manufacturing complexity have been provided so far but the very first metric is associated with the Shannon's information theory [2] related to the amount of information (in bits) in uncertainty of information system. From this approach, it is evident, that the fewer processes, machines and/or product configurations - the lower is the overall complexity of the system. Zhu et al. [3] and Desmukh et al. [4] applied and proposed entropy based measures in terms of assembly in conjunction with part types and derived their own measures to capture the process complexity in manufacturing. Suh [5] defined complexity in relation to product design through achievement of functional and design requirements. Kim et al. [6] introduced number of metrics for complexity on the basis of system components, elements and their relations. These measures cover majority of system elements but cannot be extended to other manufacturing domains except for cell production. Frizelle and Woodcock [7] defined two original types of complexity, static and dynamic currently corresponding with structural and operational complexity. Their metrics have been further applied and even developed in the works of other authors [8-11]. Other

Other authors [e.g. 12-15] discussed complexity sources in terms of manufacturing, supply chain management, machining.

\section{Methodological framework}

In the context with this objective, let us start by introduction of product configurations for the three component types, when assuming the following number of input components: number of stable components $i=2$, number of voluntary optional components $j=3$ and the number of compulsory optional components $k=2$, while only one of them must be selected $(l=1)$. This customizable assembly operation follows the Class and Sub-class of product configuration $\mathrm{CL}_{i} \mathrm{SCL}_{j}{ }_{j} \rightarrow \mathrm{CL}_{2} \mathrm{SCL}_{3}^{2}$. Then, exactly six product configurations may occur (see Fig. 1) under the assumption that the case represents single assembly node.

In a case when the same assembly node composition is a part of assembly chain (with up-stream assmebly), then the number of product configurations is a multiplication of all upstream product configurations within this assembly line.

Theoretically, for all cases of the Class $C L_{l}$, single stable component is not be counted as a product configuration. If the number of stable components $i \geq 2$, 
PRODUCT VARIETY INDUCED COMPLEXITY AND ITS MEASUREMENT

Slavomir Bednar; Vladimir Salanci

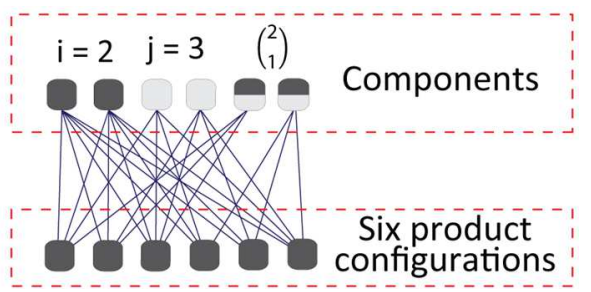

Figure 1 Single assembly model in terms of MC resulting with six product configurations

and if zero voluntary optional components are selected, we identify one product configuration for assembly of only stable components.

\subsection{Product configuration ontology}

In this section, wider description for quantification of all possible design alternatives understood as the product design space, will be treated.

This description refers to hierarchy of product ontology, as shown in Fig. 2.

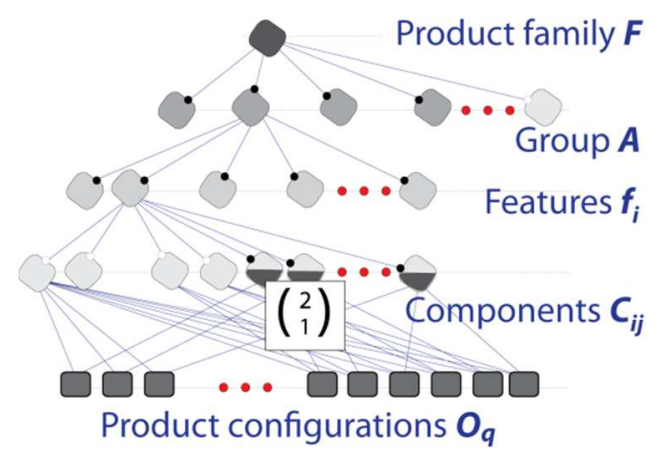

Figure 2 Product configuration ontology

To explain the hierarchy, the following working definitions can be useful. Any product activity is here considered as a compact package of products divided into features $\left\{f_{1}, \ldots f_{m}\right\}$. Feature consists of a set of components $f_{i}=\left\{C_{i 1}, C_{i 2}, \ldots, C_{i j}\right\}$. Components represent characteristics of individual feature and will be denoted as $C_{i j}$, where $j=(1, \ldots n)$ and $i=(1, \ldots m)$. Each component is linked by options (product configurations) $O_{q}=\left\{O_{1}, O_{2}, \ldots, O_{r}\right\}$, but the number of configurations depends on structure of components which can be of different nature.

\subsection{Combinatorial models for individual assembly nodes}

The purpose of determining configurations during the product development phase is to assess relative degree of service customization, since this measure expresses external perception of variety induced complexity. As it was already proved by Krus [16], value of vVIC should be reduced to minimum. In order to proceed in accordance with this statement, this sub-section aims to propose a framework for determination of all possible product configurations.

Product variety models in our approach will be represented by directed bipartite graphs. Initial nodes in such graphs are represented by components of product features and end-nodes are represented by the number of all possible product configurations (see Fig. 2).

Configuration definition process captures the requirements of both, customer and producer of the future product. For such purpose and to satisfy the MC principles, this paper proposes to adopt so called Scenarios assuming combining the three component types. We distinguish between the following situations: Scenario\#1 is the one in which only stable and voluntary component permutations play a role for consumers. Within this scenario, only combinations of available stable $C_{i j}^{S}$ and voluntary optional $C_{i j}^{V}$ components belonging to feature $f_{i}$ are counted among possible product configurations. In Scenario\#2, additional component type may appear in the definition of product composition, namely compulsory optional component $C^{C}{ }_{i j}$. This component type provides higher flexibility in specifying the future product composition. Summary scenario merging three attributes types together offers high flexibility of product composition in line with MC strategy. Final number of all available options configurations demonstrates the extent of product variability as consumers are only focused on the extent of customization offered.

The two individual Scenarios\#1-2 present a unique methodology to combine the three mostly used component types that the product, design and production engineers have to be aware of when designing new product design architecture. Appearance of the two scenarios is very frequent in the production architecture engineering. In our previous works (e.g. ([17-20]), a calculation method to obtain all possible product alternatives $\sum$ Conf within the Scenarios\#1-2 has been provided as follows:

$$
\sum \text { Conf }=\prod_{\substack{i=1 \\ j=1}}^{m}\left(\sum_{q=1}^{r} o_{q}\right)_{i_{j}}
$$

Practical importance of such measure, especially in the case of high numbers of available components, can be explained by the following example. Let us say that a structure of feature components consists of all three component types. If we had three components $C_{i j}^{V}$ and three compulsory components, where selection of at most two of them is allowed. Then, one would obtain in total 42 product configurations.

Enumeration of the final value of product options is based on the previously developed combinatorial metric of product variability (Equation 1): 
PRODUCT VARIETY INDUCED COMPLEXITY AND ITS MEASUREMENT

Slavomir Bednar; Vladimir Salanci

$$
\begin{gathered}
\sum \text { Conf }=\left(2^{j}\right) \times\left(\sum_{j=1}^{n} C_{i j}^{C}\left(\frac{k !}{l !(k-l) !}\right)\right)=2^{3} \times \\
\left(\left(\frac{3 !}{1 !(3-1) !}\right)+\left(\frac{3 !}{2 !(3-2) !}\right)\right)=8 \times(3+3)=42,
\end{gathered}
$$

where $l$ is the number of components to be chosen from a set of all components $k$ belonging to feature $f_{i}$; component $C^{C_{i j}}$ adopts the condition that $l \leq l<k$.

Current product variety in terms of MC may involve high number of features and attributes. Then, each feature can be represented by the different types of components. Products have either fixed features with voluntary attributes or they are completely customizable. The problem of such product is in the quantification of all possible design alternatives that are understood as the design space. Then, designers have to cope with future the extent of variety already in the design stage of product development. In the case of complex products, there is a lack of consistent methodological frameworks allowing to model representations of a large number of components.

\section{Combinatorial-based configuration complexity scale}

On the basis of the above-explained methodology, it is possible to create a graph showing the number of component configurations for class $C L_{2}$ based on the dependence of component configurations and the number of voluntary optional components $C^{V}{ }_{i j}$. It is evident that the number of component configurations varies only depending on the number of voluntary optional components.

As can be seen in Fig. 3(a), component class $C L_{2}$ is substantial for the creation of Configuration complexity (CC) scale. The class reaches the highest values of component configurations among all classes for the given number of initial nodes (stable and voluntary optional components). The values of component configurations from $C L_{2}$ are then considered to be the upper bound values of the scale concept. In order to define a degree of complexity, a concept of configuration complexity scale is defined, as can be seen in Fig. 3(b).

Procedure for creation of configuration scale concept consisted of the following steps:

$\circ \quad$ Generation of all possible component (product) assembly configurations;
- Defining the number of component configurations of each component class $C L$ and sub-class $S C L$;

- Analysis of the results and obtaining the upper bound values of configuration complexity scale.

Complexity degrees/levels based on total number of component configurations in case when $C L_{2}$ can be defined, as seen in Table 1.

Table 1 Complexity degrees based on summary values of product configurations

\section{The first level CC with 2-4 config.; in notation} $S C L_{1-2}$

2. The second level CC with 5-8 config.; in notation; to $\mathrm{SCL}_{3}$

3. The third level CC with 9-16 config.; in notation; to $\mathrm{SCL}_{4}$

4. The fourth level CC with 17-32 config.; in notation; to $S C L_{5}$

5. The fifth level $\mathrm{CC}$ with $33-64$ config.; in notation; to $S C L_{6}$

6. The sixth level CC with $65-128$ config.; in notation; to $\mathrm{SCL}_{7}$

7. The seventh level CC with 129-256 config.; in notation; to $\mathrm{SCL}_{8}$

8. The eighth level CC with 257-512 config.; in notation; to $S C L_{9}$

9. Etc.

Numbers in brackets are the values of component configurations for a certain number of stable and optional components.

\section{Conclusions}

This pape aimed to contribute to the theory of complexity management as it concernes with number of disciplines, such as operations research, supply chains, product portfolio, prodocution and technological complexity, variety, with aim to optimize. Moreover, Theoretical background of the problem area provides linking of the mentioned disciplines and additionally, provides solution for the management of product complexity.

Various researches, so far, dealt with generally known and derived appraoches leading to manufacturing complexity optimization, to general process optimization, such as platforming, modularization or MC in general. 
PRODUCT VARIETY INDUCED COMPLEXITY AND ITS MEASUREMENT

Slavomir Bednar; Vladimir Salanci

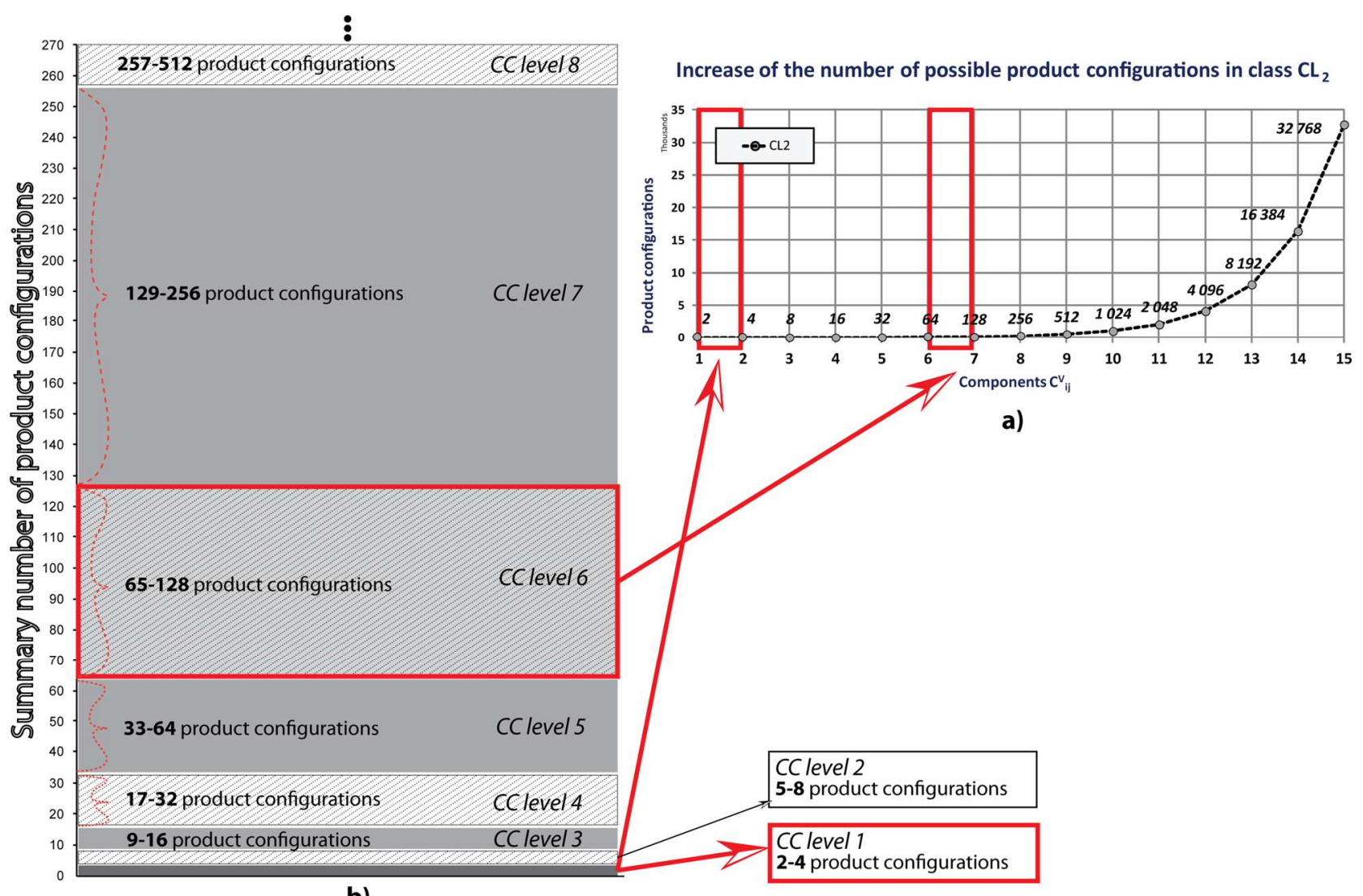

b)

Figure 3 (a) Increase of the number of configurations in $\mathrm{CL}_{2} ;($ b) Graphical representation of CC scale with defined upper bounds

None of the appraoches, so far, provided a valid or comprehensive measure of product-based complexity in terms of MC.

There is still room for future research in this area, e.g. product complexity along the product life cycle, including product design stage, production/assembly, delivery,...). Framework Scenarios \#1-2 developed under the theoretical analysis, have determined minimum preconditions for the development of conceptual framework of mass customized manufacturing (MCM). Individual building elements of the MCM have been organized into a system with roles, conditions and mutual relations among them allowing product managers to make clear easily-obtained decision for the suitable product platform variety. This way, it is possible to apply the quantification method to enumerate number of product configuratinos of any MCM model and to determine the value of the system's CC. Subsequently, it is possible to benchmark concurrent systems' CCs using the CC slace, where one of the alternative varieties may have lower level of CC than other. Such model may have lower number of product alternatives for customers but with enough demand for their products.

\section{References}

[1] WANG, H., KO, J., ZHU, X., HU, S.J.: A Complexity model for assembly supply chain and its application to configuration design, Journal of Manufacturing Science ASME Transactions, Vol. 132, pp. 5-21, 2010.

[2] SHANNON, C.E.: A mathematical theory of communication, Bell Systems Technical Journal, Vol. 27, No. 3, pp. 379-423, 1948.

[3] ZHU, X., KOREN, S.J., MARIN, S.P.: Modeling of manufacturing complexity in mixed-model assembly lines, Journal of Manufacturing Science ASME Transactions, Vol. 130, No. 5, pp. 313-334, 2008.

[4] DESMUKH, A.V., TALAVAGE, J.J., BARASH, M.M.: Complexity in manufacturing systems, part 1: Analysis of static complexity, IEEE Transactions on Engineering Management, vol. 30, No. 7, pp. 645$655,1998$.

[5] SUH, N.P.: Complexity in engineering, Annals of Manufacturing Technology, Vol. 54, No. 2, pp. 46-63, 2005.

[6] KIM, K., SHIN, Y., WU, C.: 'Complexity measures for object-oriented program based on the entropy'. Software Engineering Conference Proceedings, Asia Pacific: IEEE, pp. 127-136, 1995.

[7] FRIZELLE, G., WOODCOCK, E.: Measuring complexity as an aid to developing operational complexity, International Journal of Operations and 
PRODUCT VARIETY INDUCED COMPLEXITY AND ITS MEASUREMENT

Slavomir Bednar; Vladimir Salanci

Production Management, Vol. 15, No. 5, pp. 26-39, 1995.

[9] HAUG, A., HVAM, L., MORTENSEN, N. H.: A layout technique for class diagrams to be used in product configuration projects, Computers in Industry, Vol. 61, No. 5, pp. 409-418, 2010.

[10] KLUTH, A., JÄGER, J., SCHATZ, A., BAUERNHANSL, T.: Method for a Systematic Evaluation of Advanced Complexity Management Maturity, Procedia CIRP, Vol. 19, pp. 69-74, 2014.

[11] LUKAS, L., PLEVNY, M.: Using entropy for quantitative measurement of operational complexity of supplier-customer system: case studies, Central European Journal of Operations Research, pp. 1-17, 2015.

[12] ANISIC, Z., KRSMANOVIC, C.: Assembly initiated production as a prerequisite for mass customization and effective manufacturing, Strojniški vestnik, Vol. 54, No. 9, pp. 607-618, 2008.

[13] HUSAR, J., DUPLAKOVA, D.: Material flow planning for bearing production in digital factory, Key Engineering Materials, Vol. 669, pp. 541-550, 2016.

[14] MATISKOVA, D.: Working process optimization and minimization of production costs, Strojarstvo, Vol. 55, No. 3, pp. 231-235, 2013.

[15] FLIMEL, M.: Complex ergonomic subsystem management on workplaces from point of light climate view, Przeglad Elektrotechniczny, Vol. 87, No. 1, pp. 300-302, 2011.

[16] HRICOVA, R., BALOG, M.: Introduction of RFID system into transport and defining its model of return on investment, International Journal of Engineering Research in Africa, Vol. 18, pp. 130135, 2015.

[17] KRUS, P.: 'Design Space Configuration for Minimizing Design Information Entropy', Proceedings of the ICoRD'15, Research into Design Across Boundaries: Theory, Research Methodology, Aesthetics, Human Factors and Education, Springer India, pp. 51-60, 2015.

[18] Soltysova, Z., and S. Bednar. "Complexity Management in Terms of Mass Customized Manufacturing." Polish Journal of Management Studies 12.2 (2015): 139-49 (Original in Hungarian)

[19] BEDNAR, S., MODRAK, J.: Product variety management as a tool for successful mass customized product structure. Polish Journal of Management Studies, Vol. 12, No. 1, pp. 16-25, 2015.

[20] MODRAK, V., KRUS, P., BEDNAR, S.: Approaches to product variety management assuming configuration conflict problem, FME Transactions, Vol. 43, No. 4, pp. 271-278, 2015.

[21] MODRAK, V., MARTON, D., BEDNAR, S.: The influence of mass customization strategy on configuration complexity of assembly systems, Procedia CIRP, Vol. 33, pp. 538-543, 2015.

\section{Acknowledgement}

This paper has been supported by KEGA project no. 078TUKE-4/2015 granted by the Ministry of Education of the Slovak Republic.

\section{Review process}

Single-blind peer reviewed process by two reviewers. 